\title{
Unfaithful Glitch Propagation in Existing Binary Circuit Models
}

\author{
Matthias Függer ${ }^{1} \quad$ Thomas Nowak $^{2} \quad$ Ulrich Schmid $^{1}$ \\ ${ }^{1}$ ECS Group, TU Wien, Austria \\ ${ }^{2}$ LIX, École polytechnique, France
}

\begin{abstract}
We show that no existing continuous-time, binary value-domain model for digital circuits is able to correctly capture glitch propagation. Prominent examples of such models are based on pure delay channels (P), inertial delay channels (I), or the elaborate PID channels proposed by Bellido-Díaz et al. We accomplish our goal by considering the solvability/non-solvability border of a simple problem called Short-Pulse Filtration (SPF), which is closely related to arbitration and synchronization. On one hand, we prove that SPF is solvable in bounded time in any such model that provides channels with nonconstant delay, like I and PID. This is in opposition to the impossibility of solving bounded SPF in real (physical) circuit models. On the other hand, for binary circuit models with constant-delay channels, we prove that SPF cannot be solved even in unbounded time; again in opposition to physical circuit models. Consequently, indeed none of the binary value-domain models proposed so far (and that we are aware of) faithfully captures glitch propagation of real circuits. We finally show that these modeling mismatches do not hold for the weaker eventual SPF problem.
\end{abstract}

\section{Introduction}

Binary value-domain models that allow to model glitch propagation have always been of interest, especially in asynchronous design [22]: Pure delay channels and inertial delay channels, which propagate input pulses with some constant delay only when they exceed some minimal duration, are still the basis of most digital timing analysis approaches and tools. The tremendous advances in digital circuit technology, in particular increased speeds and reduced voltage swings, raised concerns about the accuracy of these models [3]. For example, neither pure nor inertial delay models can express the well-known phenomenon of propagating glitches that decay from stage to stage, which is particularly important for analyzing high-frequency pulse trains or oscillatory metastability [16].

At the same time, the steadily increasing complexity of contemporary digital circuits fuels the need for fast digital timing analysis techniques: Although accurate Spice models, which facilitate very precise analoglevel simulations, are usually available for those circuits, the achievable simulation times are prohibitive. Refined digital timing analysis models like the PID model proposed by Bellido-Díaz et al. [3], which is both fast and more accurate, are hence very important from a practical perspective [4].

The interest in binary models that faithfully model glitch propagation and even metastability has also been stimulated recently by the increasing importance of incorporating fault-tolerance in circuit design [7: Reduced voltage swings and smaller critical charges make circuits more susceptible to particle hits, crosstalk, and electromagnetic interference [13, 17. Since single-event transients, caused by an ionized particle hitting a reverse-biased transistor, just manifest themselves as short glitches, accurate propagation models are important for assessing soft error rates, in particular, for asynchronous circuits. After all, if system-level fault-tolerance techniques like triple modular redundancy are used for transparently masking value failures, the only remaining issue are timing failures, among which glitches are the most problematic ones.

For example, the DARTS Byzantine fault-tolerant distributed clock generation approach 12 employs standard asynchronous circuit components, like micropipelines [21, which store clock ticks received from other nodes; a new clock tick is generated when sufficiently many micropipelines are non-empty. Clearly, 
since any "wait-for-all" mechanism may deadlock in the presence of faulty components, handshaking had to be replaced by threshold logic in conjunction with some bounded delay assumptions. This way, DARTS can tolerate arbitrary behavior of Byzantine faulty nodes, except for the generation of pulses with a duration that drive the Muller C-elements of a pipeline into metastability. Analyzing the propagation of such pulses along a pipeline is thus important in order to assess the achievable resilience against such threats [11]. The situation is even worse in case of self-stabilizing algorithms [9], which must be able to recover from an arbitrary initial/error state: Neither handshaking nor any bounded delay condition can be resorted to during stabilization in an algorithm like the one presented by Dolev et al. [8]. Consequently, glitches and the possibility of metastability cannot be avoided.

As a consequence, discrete-value circuit models, analysis techniques and supporting tools for a fast but nevertheless accurate glitch and metastability propagation analysis will be a key issue in the design of future VLSI circuits. In this paper, we rigorously prove that none of the existing binary-value candidate models proposed in the past captures glitch propagation adequately. Searching for alternative models is hence an important challenge for future research on asynchronous circuits.

Detailed contributions. In Section 2, we define the Short-Pulse Filtration (SPF) problem in the physical circuit model of Marino and recall the behavior of physical circuits with respect to SPF. That is, we show that unbounded SPF is solvable with physical circuits while bounded SPF is not. The SPF problem is closely related to glitch propagation, as it is essentially the problem of building a one-shot inertial channel.

In Section 3, we present a generic binary value-domain model for digital clocked and clockless circuits, and introduce the SPF problem. Our generic model comprises zero-time logical gates interconnected by channels that encapsulate model-specific propagation delays and related decay effects. Non-zero time logical gates can be expressed by appending channels with delay at the gate's inputs and outputs. The simplest channel is a pure delay channel, which propagates its input signal with a fixed delay and without any decay, i.e., a pulse has the same duration at the channel's input and output.

In Section 4, we prove that even unbounded SPF is unsolvable when only pure, i.e., constant-delay channels are available. This is in contrast with the solvability result with physical circuits of Section 2 ,

In Section 5, we turn our attention to a generalization of constant-delay channels, termed bounded singlehistory channels, which are FIFO channels with a generalized delay function that also takes into consideration the last output transition. We distinguish between forgetful and non-forgetful single-history channels, depending on their behavior when a pulse disappears at the output due to decay effects. All existing binary models we are aware of can be expressed as single-history channels with specific delay functions: A pure delay channel (P) as either a forgetful or non-forgetful single-history channel, a classical inertial delay channel (I) as a forgetful single-history channel, and the channel model proposed by Bellido-Díaz et al. 3] (PID), which additionally has a decay component, as a non-forgetful single-history channel.

In Section [6] we prove that bounded SPF is solvable if just a single forgetful or non-forgetful singlehistory channel with non-constant delay is available. However, this is again in contradiction with the result of Section 2 showing impossibility of bounded SPF with physical circuits.

In Section 7 , we prove that weakening SPF to eventual SPF fails to witness the above modeling mismatch: Eventual SPF can by solved both with single-history and physical channels.

Fig. 1 1 summarizes our (im)possibility results.

\begin{tabular}{r|c|c|c|c|}
\cline { 2 - 5 } bounded SPF & X & $\checkmark$ & $\checkmark$ & X \\
\cline { 2 - 5 } SPF & X & $\downarrow$ & $\checkmark$ & $\checkmark$ \\
\cline { 2 - 5 } eventual SPF & $\checkmark$ & $\checkmark$ & $\checkmark$ & $\checkmark$ \\
\cline { 2 - 5 } & \multicolumn{2}{l}{ constant forgetful $\begin{array}{c}\text { non- } \\
\text { forgetful }\end{array}$}
\end{tabular}

Figure 1: Possibility $(\checkmark)$ and Impossibility (X) Results for constant, non-constant forgetful, non-const. non-forgetful, and physical physical channels. Arrows mark implications. 
Related Work. Unger 22] proposed a general technique for deriving asynchronous sequential switching circuits that can cope with unrelated input signals. It assumes signals to be binary valued, and requires the availability of combinational circuit elements, as well as pure and inertial delay channels.

Bellido-Díaz et al. 3 proposed the PID model, and justified its appropriateness both analytically and by comparing the model predictions against Spice simulation results. The results confirm very good accuracy even for such challenging scenarios as long chains of gates and ring oscillators.

Marino [15] showed that the problem of building a synchronizer can be reduced to the problem of building an inertial delay channel. The reduction circuit only makes use of combinational gates and pure delay channels in addition to inertial delay channels. Marino further shows, in a continuous value signal model, that for a set of standard designs of inertial delay channels, input pulses exist that produce outputs violating the requirements of inertial delay channels. Barros and Johnson [2] extended this work, by showing the equivalence of arbiter, synchronizer, latch, and inertial delay channels.

Marino [16] developed a general theory of metastable operation, and provided impossibility proofs for metastability-free synchronizers and arbiter circuits for several continuous-value circuit models. Branicky [5] proved the impossibility of time-unbounded deterministic and time-invariant arbiters modeled as ordinary differential equations. Mendler and Stroup [18] considered the same problem in the context of continuous automata.

Brzozowski and Ebergen [6] formally proved that, in a model that uses only binary values, it is impossible to implement Muller C-Elements (among other basic state-holding components used in (quasi) delay-insensitive designs) using only zero-time logical gates interconnected by wires without timing restrictions.

\section{Short-Pulse Filtration in Physical Systems}

In this section, we will introduce the SPF problem in the model of Marino [16] and use the classic results obtained for bistable elements to determine the solvability/impossibility border of the SPF problem for real (physical) circuits.

The model of Marino considers circuits which process signals with both continuous value domain and continuous time domain. Accordingly, we assume (normalized) signal voltages to be within $[0,1]$, and denote by $L_{0}=\left[0, l_{0}\right]$ resp. $L_{1}=\left[l_{1}, 1\right]$, with $0<l_{0}<l_{1}<1$, the signal ranges that are interpreted as logical 0 resp. logical 1 by a circuit.

A physical circuit with a single input and a single output solves Short-Pulse Filtration (SPF), if it fulfills the following requirements:

(i) If the input signal is constantly logical 0 , then so is the output signal.

(ii) There exists an input signal such that the output signal attains logical 1 at some point in time.

(iii) There exists some fixed $\varepsilon>0$ such that, if the output signal is not interpreted as logical 1 at two points in time $t$ and $t^{\prime}$ with $t^{\prime}-t<\varepsilon$, then it is not logical 1 at any time in between $t$ and $t^{\prime}$. Informally, this condition prohibits output signals that may be interpreted as pulses (see Section 3.5) with a duration less than $\varepsilon$.

A physical circuit solves bounded SPF if additionally:

(iv) There exists a time $T$ such that, if the input signal switches to logical 1 by time $t$, then the output signal value is either logical 0 or logical 1 at time $t+T$ and remains logical 0 respectively logical 1 thereafter.

We will next argue why there is no physical circuit that solves bounded SPF, but that there are physical circuits solving unbounded SPF. 


\subsection{Impossibility of Bounded SPF}

The proof is by reduction to the non-existence of a physical bistable storage element that stabilizes within bounded time in the model of Marino. A single-input bistable element is a physical circuit with a single input and a single output that fulfills properties (i) and (ii) of SPF as well as:

(iii') If the output is logical 1 at some time $t$, it also remains logical 1 at all times larger than $t$.

For a single-input bistable element stabilizing within bounded time, additionally (iv) has to hold.

The following Corollary 1 which proves the non-existence of a single-input bistable element that stabilizes within bounded time, follows immediately from Theorem 3 in [16].

Corollary 1. There is no single-input bistable element stabilizing within bounded time.

Now assume, for the sake of a contradiction, that there existed a physical circuit solving bounded SPF and consider the circuit shown in Fig. 2, with the NOR's initial output equal to 1 and the inverter's initial output equal to 0 at time $t=0$.

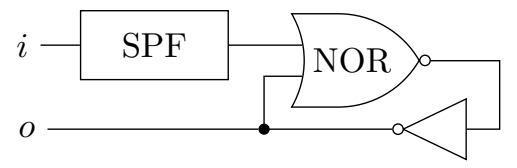

Figure 2: Building a bistable storage element from a circuit solving SPF

It is not difficult to prove that this circuit implements a single-input bistable element stabilizing within bounded time: In case the input signal $i$ is always logical 0, the SPF's output signal will always be logical 0 due to property (i) of the SPF. Thus the circuit shown in Fig. 2 will always drive a logical 0 at its output, which confirms property (i) for the bistable element.

Now let $u$ be an input pulse that makes the SPF circuit produce a logical 1 at its output. Letting $t^{\prime}$ be the first time the SPF circuit drives a logical 1 at its output, its output must remain logical 1 within $\left[t^{\prime}, t^{\prime}+\varepsilon\right]$ for some $\varepsilon>0$ due to property (iii) of the SPF. Assuming that the signal propagation delay of the NOR gate and the inverter is short enough for the inverter's output to reach a logical 1 before time $t^{\prime}+\varepsilon$, the NOR gate will subsequently drive a logical 0 on its output forever, irrespective of the output of the SPF circuit. The circuit's output signal $o$ will hence continuously remain logical 1 once it switched to logical 1 , which also confirms properties (ii) and (iii') of the bistable element.

Due to the use of a circuit solving bounded SPF in the compound circuit, we further obtain that there exists some $T>0$ such that, for any input pulse $u^{\prime}$ that switches to logical 1 by time $t$, the circuit shown in Fig. 2 produces a logical 1 by time $t+T$, a contradiction to the non-existence of a single-input bistable element stabilizing in bounded time. We hence obtain:

Theorem 1. No physical circuit solves bounded SPF.

\subsection{Possibility of Unbounded SPF}

To show the existence of a circuit solving unbounded SPF, we make use of a circuit known as a metastability filter (see, e.g., [14, p. 40]). According to Marino [16], pulses of arbitrary length may drive the internal state of every storage loop (including the one shown in Fig. 22) into a metastable region for an unbounded time. A circuit may hence produce an output signal within some region of metastable output values $\left[v_{M}^{-}, v_{M}^{+}\right] \subset[0,1]$ during an unbounded time, where the values $v_{M}^{-}$, and $v_{M}^{+}$depend on technology parameters. However, since it is possible to compute safe bounds $V_{M}^{-}$, and $V_{M}^{+}$such that $\left[v_{M}^{-}, v_{M}^{+}\right] \subset\left[V_{M}^{-}, V_{M}^{+}\right] \subset[0,1]$, a continuously valid output signal can be produced by means of a subsequent high-threshold buffer: By connecting the output $o$ of Fig. 2, ignoring the SPF block, to the input of a (high-threshold) buffer, which maps input signal values within $\left[0, B_{M}^{-}\right]$to output signal values that are logical 0 , and input values within $\left[B_{M}^{+}, 1\right]$ to output values that are logical 1, where $V_{M}^{+}<B_{M}^{-}$, we obtain a physical circuit that solves (unbounded) SPF. Hence: 
Theorem 2. There is a physical circuit that solves SPF.

\section{Binary System Model}

\subsection{Signals, Events}

We consider a binary valued signal model with continuous time, i.e., signal values are from $\mathbb{B}=\{0,1\}$ and they evolve over time $\mathcal{T}=[0, \infty)$.

A signal is a function $\mathcal{T} \rightarrow \mathbb{B}$ that does not change an infinite number of times during a finite time interval and that already has its new value at a time instant of a value transition 1

A signal transition is modeled by an event. Formally an event is a pair $e=(t, x)$ in $(\mathcal{T} \cup\{-\infty\}) \times \mathbb{B}$. We call $t$ the event's time and $x$ the event's value. We use "virtual events" at $t=-\infty$ to simplify notation when specifying initial values of channels. An event list is a (finite or infinite) sequence of events.

To every signal, there corresponds an event list $\left(e_{n}\right)=\left(t_{n}, x_{n}\right)$ with the following properties:

S1) There is always an initial event at time $-\infty$.

S2) The sequence $\left(t_{n}\right)$ of event times is strictly increasing and discrete.

S3) Values are alternating: $x_{n} \neq x_{n+1}$

Conversely, every such event list corresponds to a unique signal.

\subsection{Channels, Constant-Delay Channels}

A channel $c$ is a function mapping an input signal $s$ to an output signal $c(s)$.

The simplest class of channels is the class of (positive) constant-delay channels. A constant-delay channel $c$ with delay parameter $\delta>0$ and initial value $x \in \mathbb{B}$ produces at its output the input signal delayed by $\delta$, i.e.,

$$
c(s)(t)= \begin{cases}x & \text { if } t<\delta \\ s(t-\delta) & \text { if } t \geq \delta .\end{cases}
$$

Note that a physical realization of a constant-delay channel with initial value $x$ requires a multiplexer, which supplies the constant-delay channel's input with the initial value $x$ during $(-\infty, 0)$ and switches to the actual input $s$ at reset time 0 .

\subsection{Circuits}

Circuits are obtained by interconnecting a set of input ports and a set of output ports, forming the external interface of a circuit, and a set of combinational gates via channels. We constrain the way components are interconnected in a natural way, by requiring that input ports are attached to one or more channel inputs only $(\mathrm{C} 4)$, and that both output ports and gate inputs are attached to just one channel's output (C5, C6); the latter prevents channel outputs driving against each other.

Formally, a circuit is a tuple $C=(G, I, O, c, n)$, where

C1) $G$ is a directed graph whose vertex set can be partitioned as $I \cup O \cup B$.

C2) Every vertex $b$ in $B$ ((Boolean) gate) is assigned a Boolean function $\mathbb{B}^{d_{b}} \rightarrow \mathbb{B}$, where $d_{b}$ is the in-degree, i.e., the number of incoming neighbors, of $b$. By a slight abuse of notation, $b$ also denotes the Boolean function assigned to $b$.

\footnotetext{
${ }^{1}$ The requirement that a signal already has its new value when changing values is merely a convention. On the other hand, the requirement that it only changes a finite number of times during a finite time interval is fundamental to our model and, thus, our results.
} 
C3) $c$ is a function that maps every edge $(u, v)$ in $G$ to its corresponding channel $c_{u, v}$.

C4) Every vertex $v \in I$ (input ports) has in-degree $d_{v}=0$.

C5) Every vertex $v \in O$ (output ports) has in-degree $d_{v}=1$.

C6) $n$ is a function that maps every vertex $v$ in $G$ to a linearly ordered subset $n_{v}=\left\{v_{1}, \ldots, v_{d_{v}}\right\}$ of its in-neighbor vertices in $G$, i.e., where edge $\left(v_{i}, v\right)$ for $i=1$ up to $v$ 's in-degree $d_{v}$ is in $G$.

Note that there are also zero-input Boolean gates 0 and 1 that represent constant signal values 0 and 1 .

\subsection{Executions}

An execution of circuit $C$ is an assignment of signals to vertices that respects the channel functions and Boolean gate functions.

Formally, an execution of circuit $C$ is a collection of signals $s_{v}$ for all vertices $v$ of $C$ such that the following properties hold: If $i$ is an input port, then there are no restrictions on $s_{i}$. If $o$ is an output port, then $s_{o}=c_{v, o}\left(s_{v}\right)$ where $v$ is the unique incoming neighbor of $o$ and $c_{v, o}$ the channel representing edge $(v, o)$. Let now $b$ be a Boolean gate with $d$ incoming neighbors $v_{1}, v_{2}, \ldots, v_{d}$, ordered according to $n_{b}$. We then apply, for each incoming edge $\left(v_{k}, b\right)$, the channel $c_{v_{k}, b}$ to signal $s_{v_{k}}$ and check that the signal value $s_{b}(t)$ is the gate's Boolean combination of these incoming signals at time $t$. That is, $s_{b}(t)=b\left(c_{v_{1}, b}\left(s_{v_{1}}\right)(t), \ldots, c_{v_{d}, b}\left(s_{v_{d}}\right)(t)\right)$ for all $t \in \mathcal{T}$.

Not all circuits necessarily do have executions. For example, the circuit comprising a single inverter gate whose output is fed back to its input via the "mirror channel" $c$ with $c(s)=s$ for all signals $s$ does not have an execution. Whenever we introduce a circuit for a possibility result, we will thus make sure that it allows for a unique execution once the input signals are fixed. In case of constant-delay channels, this is always the case (see Lemma 2).

\subsection{Short-Pulse Filtration}

A pulse $p$ of length $\Delta>0$ at time $T$ is a signal of the form

$$
p(t)= \begin{cases}0 & \text { if } t<T \text { or } t \geq T+\Delta \\ 1 & \text { if } T \leq t<T+\Delta .\end{cases}
$$

A signal contains a pulse of length $\Delta>0$ at time $T$ if its event list contains the two consecutive events $(T, 1)$ and $(T+\Delta, 0)$.

A circuit solves Short-Pulse Filtration (SPF) if it fulfills the following conditions:

F1) It has exactly one input port $i$ and exactly one output port $o$.

F2) For every pulse $p$, there exists an execution that has $p$ as the input signal (i.e., $s_{i}=p$ ). (Well-formedness)

F3) In all executions, if the input signal is constant zero, then so is the output signal. (No generation)

F4) There exist a pulse $p$ such that, in all executions with $p$ as the input signal, the output signal is not the constant zero signal. (Nontriviality)

F5) There exists an $\varepsilon>0$ such that, in all executions, the output signal does not contain a pulse of length less than $\varepsilon$. (No short pulses)

A circuit solves bounded SPF if additionally the following condition holds:

F6) There exists a $K>0$ such that, in all executions with a pulse as the input signal whose last event is at time $T$, the output signal does not change anymore after time $T+K$. (Bounded stabilization time) 
A circuit solves eventual SPF if conditions (F1)-(F4) and the following condition hold:

F5e) There exists an $\varepsilon>0$ and a $K>0$ such that, in all executions with a pulse at time $T$ as the input signal, the output signal does not contain a pulse of length less than $\varepsilon$ after time $T+K$. (Eventually no short pulses)

\section{Unsolvability of Short-Pulse Filtration with Constant-Delay Chan- nels}

In this section, we show that no circuit whose channels are all positive constant-delay channels solves SPF. The idea of the proof is to exploit the fact that the value of the output signal of the circuit at each time $t$ only depends on a finite number of values of the input signal at times $t^{\prime}$ between 0 and $t$.

Calling each such time $t^{\prime}$ a measure point for time $t$, we show that indeed only a finite number of measure points exists for time $t$, i.e., the circuit cannot distinguish two different input signals that do not differ in the input signal values at the measure points for time $t$ : For both such input signals, the output signal must

have the same value at time $t$. Combining that indistinguishability result with a shifting argument of the input signal allows us to construct an arbitrary short pulse at the output of the circuit, a contradiction to property (F5) of Short-Pulse Filtration.

\subsection{Dependence Graphs}

For each constant-delay circuit with a single input port and a single output port, we introduce its dependence graph, which describes the way the output signals may depend on the input signals.

Let $C=(G, I, O, c, m)$ be a circuit with constant-delay channels, a single input port $i$, and a single output port $o$. For every channel $c_{u, v}$ of $C$, denote by $\delta(u, v)$ its delay parameter $\delta$ and by $x(u, v)$ its initial value. The dependence graph $D G(t)$ of $C$ at time $t$ is a directed graph with vertices $(v, \tau)$, where $v$ is a vertex in $G$ and $\tau$ a time. It is defined as follows:

- The pair $(o, 0)$ is a vertex of $D G(t)$.

- If $(v, \tau)$ is a vertex of $D G(t)$ and $(u, v)$ is an edge in $G$ such that $\tau+\delta(u, v) \leq t$, then the pair $(u, \tau+\delta(u, v))$ is also a vertex of $D G(t)$ and there is an edge in $D G(t)$ from $(u, \tau+\delta(u, v))$ to $(v, \tau)$.

- If $(v, \tau)$ is a vertex of $D G(t)$ and $(u, v)$ is an edge in $G$ such that $\tau+\delta(u, v)>t$, then $c_{u, v}$ 's initial value $x(u, v)$ is a vertex of $D G(t)$ and there is an edge in $D G(t)$ from $x(u, v)$ to $(v, \tau)$.

Because all $\delta(u, v)$ are strictly positive, the dependence graphs are finite and acyclic. A vertex of $D G(t)$ without incoming neighbors is a leaf, all others intermediate vertices. A vertex of the form $(i, \tau)$, with $i \in I$, is an input leaf and we call the time $t-\tau$ the corresponding measure point for time $t$. If $D G(t)=D G(\tilde{t})$, then the measure points for $t$ are exactly the measure points for $\tilde{t}$ shifted by the difference $t-\tilde{t}$. All leaves of $D G(t)$ are either input leaves or elements of $\mathbb{B}$ (initial values of channels).

$$
i \stackrel{\substack{\delta=1 \\
x=0}}{\substack{\delta=2 \\
x=0}} \cdot \begin{aligned}
& \delta=1 \\
& x=0
\end{aligned}
$$

Figure 3: Example circuit

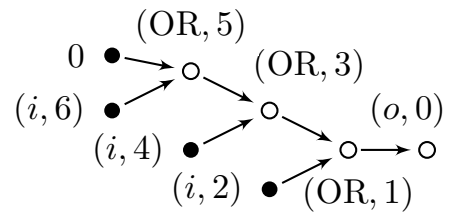

Figure 4: Example dependence graph $D G(6)$

As an example, consider the circuit shown in Fig. 3. The dependence graph $D G(6)$ is shown in Fig. 4. Leaves are depicted as filled nodes, while intermediate nodes are empty. From the construction of the graph, 
we immediately see that in each execution the output signal value $s_{o}(6)$ only depends on the (input) signal values $s_{i}(4), s_{i}(2)$, and $s_{i}(0)$. Thus, in particular, $s_{o}(6)$ is the same for both input signals depicted in Fig. 5

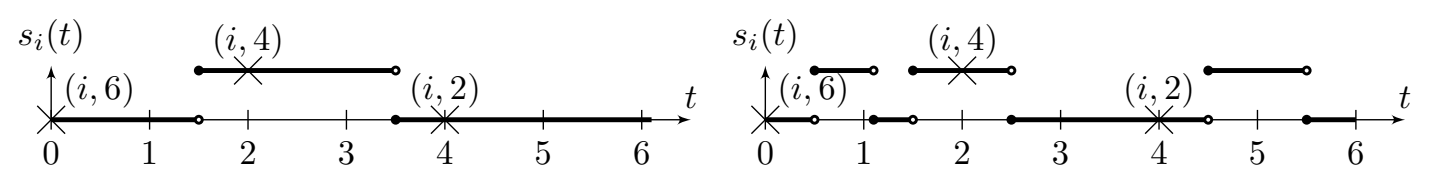

Figure 5: Input pulses with measure points $(\times)$, labeled with the corresponding input leaf names.

Generalizing the observations from the example, we thus observe:

Lemma 1. The value of the output signal at time $t$ only depends on the values of the input signal at the measure points for time $t$, according to $D G(t)$.

Furthermore, if $D G(t)=D G(\tilde{t})$ and the values of input signals $s_{i}$ and $\tilde{s}_{i}$ coincide at the respective measure points for $t$ and $\tilde{t}$, then the respective output signals fulfill $s_{o}(t)=\tilde{s}_{o}(\tilde{t})$.

Proof. For a path $\pi$ in $G$, denote by $\delta(\pi)$ the sum of delays $\delta(u, v)$ over all edges $(u, v)$ of $\pi$. For every vertex $v$ of $G$ and every time $t \in \mathcal{T}$, let $\mathcal{P}(\rightarrow y, t)$ be the set of maximum length paths $\pi$ ending in $v$ such that $\delta(\pi) \leq t$.

It is clear, by iterating Eq. (1), that the value of $s_{v}(t)$ is uniquely determined by the collection of values $s_{u}(t-\delta(\pi))$ where $u$ is the start vertex of $\pi \in \mathcal{P}(\rightarrow v, t)$. Moreover, by maximality of $\pi$, if $u \neq i$, then $s_{u}(t-\delta(\pi))$ only depends on the initial values of channels of incoming edges to $u$. Hence $s_{v}(t)$ is uniquely determined by the collection of values $s_{i}(t-\delta(\pi))$ where $\pi \in \mathcal{P}(\rightarrow y, t)$ starts at $i$. This holds in particular for $v=o$.

This lemma has as an immediate conseqeuence our remark at the end of Section 3.4:

Lemma 2. If $C$ is a circuit with only constant-delay channels, then for all assignments of input signals $\left(s_{i}\right)_{i \in I}$ there exists a unique execution of $C$ extending this assignment.

Due to the fact that there are only finitely many measure points for a given time $t$, they are discrete and hence there is always a small margin until a new measure point appears:

Lemma 3. For every $t \in \mathcal{T}$, there exists an $\varepsilon>0$ such that $D G(t)=D G\left(t+\varepsilon^{\prime}\right)$ for all $0 \leq \varepsilon^{\prime} \leq \varepsilon$.

Proof. Let $\varepsilon>0$ be strictly smaller than all positive values of the form $\delta(u, v)+\tau-t$ where $(v, \tau)$ is an intermediate vertex of $D G(t)$ and $(u, v)$ is an edge in $G$. If no such intermediate vertex or edge exists, choose $\varepsilon>0$ arbitrarily.

Let $(v, \tau)$ be an intermediate vertex of $D G(t)$ and $(u, v)$ be an edge in $G$. If $t+\varepsilon-\tau<\delta(u, v)$, then clearly $t-\tau<\delta(u, v)$, because $\varepsilon>0$. On the other hand, if $t-\tau<\delta(u, v)$, then $\delta(u, v)+\tau-t$ is positive and hence $\delta(u, v)>t+\varepsilon-\tau$ by choice of $\varepsilon$. Thus, the conditions $t-\tau<\delta(u, v)$ and $t+\varepsilon-\tau<\delta(u, v)$ are equivalent. This shows that the two dependence graphs $D G(t)$ and $D G(t+\varepsilon)$ and hence all dependence graphs in between are equal.

\subsection{Unsolvability Proof}

Assume by contradiction that $C$ solves SPF. By the nontriviality property (F4), there exists an input pulse such that the corresponding output signal is non-zero, i.e., there exists an input pulse of some length and a time $t$ such that the corresponding output signal's value at time $t$ is 1 .

By Lemma 3, there exists an $\varepsilon>0$ such that $D G(t)=D G(t+\varepsilon)$. We may choose $\varepsilon$ arbitrarily small, in particular strictly smaller than all differences of distinct measure points for time $t$.

Clearly, $D G(\tilde{t})=D G(t)$ for all times $\tilde{t}$ between $t$ and $t+\varepsilon$, in particular, for $\tilde{t}=t+\varepsilon / 2$. Denote by $\Delta$ the infimum of input pulse lengths (where all pulses start at the same time) such that the corresponding 


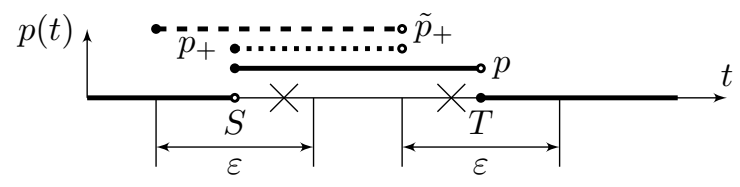

Figure 6: Input pulse $p$, together with its derived pulses $p_{+}$and $\tilde{p}_{+}$, and measure points for time $\tilde{t}$.

output signal's value at time $\tilde{t}$ is 1 . This infimum is finite by the choice of $t$ and $\tilde{t}$. There hence exists an input pulse $p$ with the above property of length at most $\Delta+\varepsilon / 4$. We show that its corresponding output signal $s_{p}$ contains a pulse of length strictly less than $\varepsilon$, in contradiction to the no short pulses property (F5).

Denote by $S$ the time of $p$ 's upwards and by $T$ the time of $p$ 's downwards transition. Now let $p_{+}$be the pulse whose upwards transition is at time $S$ and whose downwards transition is at time $T-\varepsilon / 2$. If $S \geq T-\varepsilon / 2$, then let $p_{+}$be the zero signal instead. The length of $p_{+}$is either strictly less than $\Delta$ or it is the zero signal. Hence, by the definition of the no-generation property (F3), its corresponding output signal's value at time $\tilde{t}$ is 0 . This implies that there exists a measure point for time $\tilde{t}$ within $[T-\varepsilon / 2, T)$, because $p$ and $p_{+}$coincide everywhere else (see marked measure point on the right in Fig. 6).

Because we chose $\varepsilon$ to be smaller than all differences of distinct measure points for time $t$ (and hence also for time $\tilde{t})$, we see that there is no measure point for $\tilde{t}$ in the interval $[T, T+\varepsilon / 2$ ).

Likewise, by defining $p_{-}$as the pulse with upwards transition at time $S+\varepsilon / 2$ and downwards transition at time $T$, we infer that there is one measure point for time $\tilde{t}$ in the interval $[S, S+\varepsilon / 2)$ and there is no measure point for $\tilde{t}$ in the interval $[S-\varepsilon / 2, S$ ) (see Fig. 6).

Now consider the pulse $\tilde{p}_{+}$generated by shifting pulse $p$ into the past by $\varepsilon / 2$, i.e., $\tilde{p}_{+}$'s upwards transition is at time $S-\varepsilon / 2$ and its downwards transition is at $T-\varepsilon / 2$. Because $\tilde{p}_{+}$coincides with $p_{+}$at all measure points for $\tilde{t}$, the output signal $s^{\tilde{p}_{+}}$corresponding to $\tilde{p}_{+}$has value 0 at time $\tilde{t}$. Because $D G(\tilde{t})=D G(\tilde{t}+\varepsilon / 2)$, the second part of Lemma 1 shows that $s^{\tilde{p}+}(\tilde{t}+\varepsilon / 2)=0$.

Likewise, by considering $p$ shifted into the future by $\varepsilon / 2$, we see that also $s^{\tilde{p}+}(\tilde{t}-\varepsilon / 2)=0$. But because $s^{p}(\tilde{t})=1$, this shows that the output signal $s^{p}$ contains a pulse of length strictly less than $\varepsilon$. Since $\varepsilon$ can be chosen arbitrarily small, this concludes the proof.

\section{Bounded Single-History Channels}

This section formally introduces the notion of bounded single-history channels in the binary circuit model. They are a generalization of constant-delay channels that cover all existing channel models for binary circuit models we are aware of.

Intuitively, a bounded single-history channel propagates each event, occurring at time $t$, of the input signal to an event at the output happening after some bounded output-to-input delay $\delta(T)$, which depends on the input-to-previous-output delay $T=t-t^{\prime}$. Note that $T$ is positive if the channel delay is short compared to the input signal transition times, and negative otherwise. Fig. 7 illustrates this relation and the involved delays. In case FIFO order would be invalidated, i.e., $t+\delta(T) \leq t^{\prime}$, such that the next output event would not occur after the previous one, both events annihilate.

There exist two variants of bounded single-history channels in the literature, depending on whether the time of an annihilated event is remembered or not. We dub these two variants forgetful and non-forgetful bounded single-history channels, which we both formally define below. At the end of this section, we give a list of channel models that are special cases of our definition of bounded single-history channels.

Formally, a bounded single-history channel $c$ is characterized by an initial value $x \in \mathbb{B}$, a nondecreasing delay function $\delta: \mathbb{R} \rightarrow \mathbb{R}$ such that $\delta(\infty)=\lim _{T \rightarrow \infty} \delta(T)$ is finite and positive, and the fact whether it is forgetful or not. In the rest of the paper, we will drop the qualifier "bounded" when referring to bounded single-history channels. We detail the channel behavior in the next two subsections. 


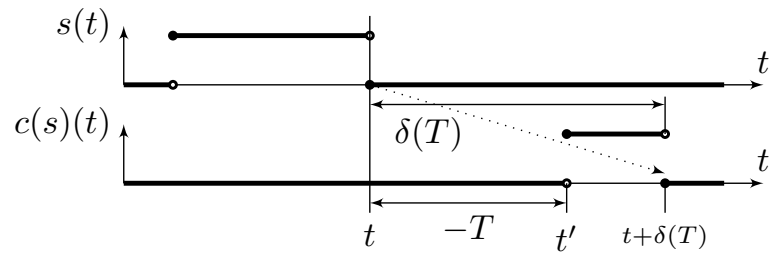

Figure 7: Input/output signal of a bounded single-history channel, involving the input-to-previous-output delay $T$ and the resulting output-to-input delay $\delta(T)$.

\subsection{Forgetful Single-History Channels}

This class of channels includes the classical inertial delay channels as used, for example, in VHDL simulators [1.

Their behavior is defined by the following algorithm: Let $s$ be a signal. In case the channel's initial value $x$ is equal to the initial value of $s$, or there is an event at time 0 in the event list of $s$, let the channel's input list $\left(\left(t_{n}, x_{n}\right)\right)_{n}$ be the event list of $s$. Otherwise, let the channel's input list be the event list of $s$ with an additional event at time 0 and value equal to the initial value of $s$. The algorithm iterates the input list and updates the output list, which will define the channel's output signal $c(s)$.

Initially, let $(-\infty, x)$ be the sole element of the output list. In its $n$th iteration the algorithm considers input event $\left(t_{n}, x_{n}\right)$ and modifies the output list accordingly:

1. Denote by $\left(t_{n}^{\prime}, x_{n}^{\prime}\right)$ the last event in the output list. If $x_{n}=x_{n}^{\prime}$, then input event $\left(t_{n}, x_{n}\right)$ has no effect: Proceed to the $(n+1)$ th iteration.

2. Otherwise, let $T_{n}=t_{n}-t_{n}^{\prime}$ be the difference of input and previous-output event times 2

If $t_{n}+\delta\left(T_{n}\right)>t_{n}^{\prime}$, then add the event $\left(t_{n}+\delta\left(T_{n}\right), x_{n}\right)$ to the output list.

If $t_{n}+\delta\left(T_{n}\right) \leq t_{n}^{\prime}$, then delete the event $\left(t_{n}^{\prime}, x_{n}^{\prime}\right)$ from the output list.

Note that the output sequence's first event is always $(-\infty, x)$, all other events have positive times (since $\delta(\infty)>0)$, its sequence of event times is strictly increasing, and its sequence of values is alternating.

If the input list is finite, the algorithm halts. If not, the output sequence nonetheless stabilizes in the sense that, for every time $t$, there exists some $N$ such that all iterations with $n \geq N$ make no changes to the output sequence at times $\leq t$. The next lemma (Lemma 4) proves this property and makes the limit output list as $n$ tends to infinity well-defined. So, even if the input list is infinite, there exists a well-defined (infinite) output list $S$ that is the result of the described algorithm. The channel's output signal $c(s)$ is then defined by event list $S$ :

Definition 1. For input signal $s$, the output signal $c(s)$ of the forgetful single-history channel $c$ is the signal whose event list is the list $S$ as defined by the above algorithm.

Lemma 4. Denote by $S_{n}$ the output list after the nth iteration of the forgetful channel algorithm, and by $S_{n} \mid t$ its restriction to the events at times at most $t$. For all $t$ there exists an $N$ such that $S_{n} \mid t$ is constant for all $n \geq N$.

Proof. The lemma is trivial if the input list is finite, so we assume it to be infinite.

Because the sequence of input event times $\left(t_{n}\right)$ tends to infinity, there exists an $N$ such that

$$
t_{N} \geq \max (t, t-\delta(-\delta(\infty))) .
$$

We show by induction that $S_{n}\left|t=S_{N}\right| t$ for all $n \geq N$. This is trivial for $n=N$, so let $n>N$. Then $t_{n}>t_{N}$.

\footnotetext{
${ }^{2}$ Note that $T_{n}=\infty$ is possible. In this case $\delta\left(T_{n}\right)=\delta(\infty)=\lim _{T \rightarrow \infty} \delta(T)$, which is finite by assumption.
} 
Let $\left(t_{n}^{\prime}, x_{n}^{\prime}\right)$ be the last element in $S_{n-1}$, and $T_{n}=t_{n}-t_{n}^{\prime}$. The case $x_{n}=x_{n}^{\prime}$ is trivial, so let $x_{n} \neq x_{n}^{\prime}$. We distinguish two cases, depending on whether $\delta\left(T_{n}\right)>-T_{n}$ or not:

Case 1: $\delta\left(T_{n}\right)>-T_{n}$. Because $\delta$ is nondecreasing, $\delta\left(T_{n}\right) \leq \delta(\infty)$, and hence $T_{n}>-\delta(\infty)$ and also $\delta\left(T_{n}\right) \geq \delta(-\delta(\infty))$. This implies $t_{n}+\delta\left(T_{n}\right)>t_{N}+\delta(-\delta(\infty)) \geq t$ by using (3). Hence $S_{n}\left|t=S_{n-1}\right| t=S_{N} \mid t$ by the induction hypothesis.

Case 2: $\delta\left(T_{n}\right) \leq-T_{n}$. We show that $t_{n}^{\prime}>t$ by contradiction: Let $t_{n}^{\prime} \leq t$. Then $T_{n}=t_{n}-t_{n}^{\prime}>t_{N}-t \geq 0$, by using (3). From $\delta(\infty)>0$, we thus obtain $T_{n}>-\delta(\infty)$. Hence $\delta\left(T_{n}\right) \geq \delta(-\delta(\infty))$ by monotonicity of $\delta$. By assumption, $\delta(-\delta(\infty)) \leq \delta\left(T_{n}\right) \leq-T_{n}=t_{n}^{\prime}-t_{n}$, which implies $t_{n} \leq t_{n}^{\prime}-\delta(-\delta(\infty))$, i.e., $t_{N}<t-\delta(-\delta(\infty))$. This is a contradiction to (3), which shows that $t_{n}^{\prime}>t$. Hence $S_{n}\left|t=S_{n-1}\right| t=S_{N} \mid t$ by the induction hypothesis.

\section{$5.2 \quad$ Non-Forgetful Single-History Channels}

The PID channel introduced by Bellido-Díaz et al. 3 is not covered by the above forgetful single-history channels, since it has been designed to reasonably match analog RC waveforms: Analog signals like exponential functions do not "forget" sub-threshold pulses. Hence, they cannot be modeled via delay functions $\delta(T)$ that depend on the input-to-previous output delay $T$. To also cover the PID model, we hence introduce non-forgetful single-history channels, the delay function of which may also depend on the last annihilated event.

The output-eventlist generation algorithm for non-forgetful channels thus maintains an additional variable $r$, which, in each iteration, contains the time of the potential output event considered in the last iteration. Note that this approach was already used in the PID-channel-model by Bellido-Díaz et al. 3, Fig. 13]. Similar to the forgetful case, it determines the output signal $c(s)$ of a non-forgetful single-history channel $c$, given input signal $s$ with input event list $\left(\left(t_{n}, x_{n}\right)\right)_{n}$ as follows:

Initially, the output list contains the sole element $(-\infty, x)$ and $r=r_{-1}=-\infty$. In its $n$th iteration, the algorithm considers input event $\left(t_{n}, x_{n}\right)$ and modifies the output list accordingly:

1. Denote by $\left(t_{n}^{\prime}, x_{n}^{\prime}\right)$ the last event in the output list. If $x_{n}=x_{n}^{\prime}$, then input event $\left(t_{n}, x_{n}\right)$ has no effect: Proceed to the $(n+1)$ th iteration.

2. Otherwise, let $T_{n}=t_{n}-r_{n-1}$ be the difference of input and most recent potential output event times and set $r_{n}=t_{n}+\delta\left(T_{n}\right)$.

If $t_{n}+\delta\left(T_{n}\right)>r_{n-1}$, then add the event $\left(t_{n}+\delta\left(T_{n}\right), x_{n}\right)$ to the output list.

If $t_{n}+\delta\left(T_{n}\right) \leq r_{n-1}$, then delete the event $\left(t_{n}^{\prime}, x_{n}^{\prime}\right)$ from the output list.

We first show that if event $\left(t_{n}^{\prime}, x_{n}^{\prime}\right)$ is deleted in the $n$th iteration, then $r_{n-1}=t_{n}^{\prime}$.

Proof. Assume by contradiction that this is not the case, and let $n$ be the first iteration where the statement is violated. Then it must hold that $n \geq 2$, as in iteration $n-2$ some event $\left(\tau, x_{n-2}\right)$ must have been added to the output list that was deleted in iteration $n-1$, due to $\tau^{\prime}=t_{n-1}+\delta\left(T_{n-1}\right) \leq r_{n-2}=\tau$. Furthermore, in iteration $n$, our assumption of deleting some event with a time different from $r_{n-1}=\tau^{\prime}$ implies $\tau^{\prime \prime}=$ $t_{n}+\delta\left(T_{n}\right) \leq \tau^{\prime}$. However, from $t_{n-1}<t_{n}, \tau \geq \tau^{\prime}$ and monotonicity of $\delta, t_{n-1}+\delta\left(t_{n-1}-\tau\right)<t+\delta\left(t-\tau^{\prime}\right)$, i.e., $\tau^{\prime}<\tau^{\prime \prime}$, which provides the required contradiction.

Thus, an event is either deleted in the next iteration, or never deleted. The output sequence's first event $(-\infty, x)$ is obviously never deleted.

By analogous arguments, one can show that the sequence of event times is strictly increasing, with an alternating sequence of values. Unlike in the case of forgetful channels, however, the eventlist generation algorithm may produce events with finite negative times that will be removed from the final output. In case the input list is finite, the algorithm clearly halts. If not, we again have the same stabilization property as for forgetful single-history channels, which we will provide in Lemma 5 below. Thus the algorithm's final output list $S$ is again well-defined and we can define: 
Definition 2. For input signal $s$, the output signal $c(s)$ of the forgetful single-history channel $c$ is the signal whose event list is the list $S$ as defined by the above algorithm. after deleting all events with finite negative times and the first non-negative time event if its value is equal to the channel's initial value $x$.

Lemma 5. Denote by $S_{n}$ the output list after the $n$-th iteration of the forgetful channel algorithm, and by $S_{n} \mid t$ its restriction to the events at times at most $t$. For all $t$, there exists an $N$ such that $S_{n} \mid t$ is constant for all $n \geq N$.

Proof. The lemma follows from the fact that an event can only be deleted one iteration after it was added to the output list, and the fact that in each iteration $n, T_{n}>-\delta(\infty)$ and thus $t_{n}+\delta\left(T_{n}\right)$ is lower bounded by $t_{n}+\lim _{t \rightarrow 0^{+}} \delta(-\delta(\infty)+t)$.

\subsection{Examples of Single-History Channels}

Below, we summarize how the existing binary-value models are mapped to our single-history channels:

1) A classic pure-delay channel is a single-history channel whose delay function $\delta$ is constant and positive. The behavior of a pure-delay channel does not depend on whether it is forgetful or not.

2) An inertial channel is a forgetful single-history channel whose delay function $\delta$ is of the form

$$
\delta(T)= \begin{cases}\delta_{0} & \text { if } T>T_{0} \\ -T_{0} & \text { if } T \leq T_{0}\end{cases}
$$

for parameters $\delta_{0}>0$ and $T_{0}>-\delta_{0}$. An inertial channel filters an incoming pulse if and only if its pulse length is less or equal to $T_{0}+\delta_{0}$; otherwise, it is forwarded with delay $\delta_{0}$.

3) The PID-channels of Bellido-Díaz et al. [3] are non-forgetful with delay function

$$
\delta(T)=t_{p 0} \cdot\left(1-e^{-\left(T-T_{0}\right) / \tau}\right)
$$

for certain (measured) positive parameters $t_{p 0}, \tau$, and $T_{0}$. Note that $\delta\left(T_{0}\right)=0, \lim _{t \rightarrow \infty} \delta(T)=t_{p 0}$, and $\left.\frac{d \delta(T)}{d T}\right|_{T=0}=t_{p 0} / \tau$ here.

\section{Bounded Short-Pulse Filtration with One Non-Constant Delay Channel}

In this section we prove that bounded SPF is solvable as soon as there is a single non-constant-delay singlehistory channel available. More specifically, we show that, given a single-history channel with non-constant delay, there exists a circuit that uses only constant-delay channels apart from the given non-constant channel that solves bounded SPF. Different circuits, and hence proofs, are used in for different types of channels.

For a single-history channel with delay function $\delta$, let $\delta_{\infty}=\delta(\infty)=\lim _{t \rightarrow \infty} \delta(t)$ with $0<\delta_{\infty}<\infty$. The right limit of $\delta$ at $-\delta_{\infty}$ is denoted by $\delta_{\text {inf }}=\lim _{t \rightarrow 0^{+}} \delta\left(-\delta_{\infty}+t\right)$; note that $\delta_{\text {inf }}=-\infty$ is allowed here.

In the rest of this section, let $c^{*}$ be a single-history channel that is not a constant-delay channel as defined in Section 3.2. This is equivalent to saying that its delay function $\delta$ is non-constant for $T>-\delta_{\infty}$, because $T_{n}>-\delta_{\infty}$ in every step of the channel algorithm:

Lemma 6. A single-history channel with delay function $\delta$ is a constant-delay channel if and only if $\delta$ is constant in the open interval $\left(-\delta_{\infty}, \infty\right)$.

Note that $\delta_{\text {inf }}<\delta_{\infty}$ in case of a non-constant delay channel. From the fact that $-\delta_{\infty}<T_{n} \leq \infty$ in every step of the channel algorithm, we also obtain:

Lemma 7. All events in the event list of a single-history channel's input signal are delayed by times within $\left[\delta_{\text {inf }}, \delta_{\infty}\right]$. 


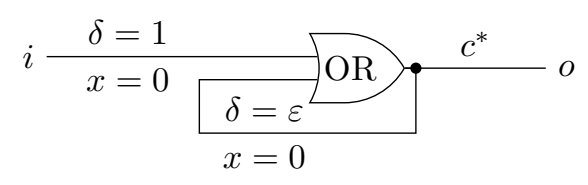

Figure 8: Circuit $C_{\mathrm{ff}}$.

\subsection{Forgetful Channels}

In this subsection, assume that $c^{*}$ is forgetful. Consider circuit $C_{\mathrm{ff}}$ depicted in Fig. 8, which contains channel $c^{*}$ as well as two constant-delay channels. For the moment assume that the initial value of $c^{*}$ is 0 . We will show at the end of this subsection that bounded SPF is also solvable with $c^{*}$ if its initial value is 1 .

It remains to describe how to choose delay parameter $\varepsilon>0$. We will show in the following that for each non-constant-delay forgetful single-history channel $c$ there exists a $\gamma(c)>0$ such that $c(s)$ is the zero signal whenever $s$ is a pulse of length less than $\gamma(c)$. More generally we will show that, if signal $s$ does not contain pulses of length greater or equal to $\gamma(c)$, then $c(s)$ is the zero signal. We then choose $0<\varepsilon<\gamma\left(c^{*}\right)$ for the delay parameter $\varepsilon$ in circuit $C_{\mathrm{ff}}$.

If the input signal of circuit $C_{\mathrm{ff}}$ is a pulse of length at least $\varepsilon$, then the signal $s_{O R}$ at the OR gate is eventually stable 1 because of the $\varepsilon$-delay feedback loop, and hence the circuit's output signal is eventually stable 1 . If the circuit's input signal is a pulse of length $\Delta<\varepsilon$, then $s_{O R}$ only contains pulses of length $\Delta<$ $\gamma\left(c^{*}\right)$, from which it follows that the circuit's output signal is zero.

Let $\delta$ be the delay function of a single-history channel $c$. We define:

$$
\gamma(c)=\inf \left\{\Delta>0 \mid \Delta-\delta_{\infty}+\delta\left(\Delta-\delta_{\infty}\right)>0\right\}
$$

We will prove $\gamma\left(c^{*}\right)>0$ in Lemma 9. Before characterizing the non-constant-delay channels as those $c$ with $\gamma(c)>0$, we need a preliminary lemma on pulse-filtration properties of non-constant-delay channels.

Lemma 8. Let $c$ be a non-constant-delay single-history channel with initial value 0 . If $s$ is a pulse of length less than $\gamma(c)$, then $c(s)$ is zero.

Proof. The event list of $s$ consists of two events $(S, 1)$ and $(T, 0)$, possibly preceded by an additional event $(0,0)$, depending on whether $S=0$ or $S>0$. Because the initial value of $c$ is 0 , we may assume without loss of generality that the sequence consists of only these two events.

After iteration $n=0$ of the channel-defining algorithm, the output list is equal to $\left((-\infty, 0),\left(S+\delta_{\infty}, 1\right)\right)$. Hence, in iteration $n=1$,

$$
T_{1}=T-S-\delta_{\infty}<\gamma(c)-\delta_{\infty}
$$

i.e., $T_{1}+\delta_{\infty}<\gamma(c)$. By definition of $\gamma(c)$, this implies

$$
\left(T_{1}+\delta_{\infty}\right)-\delta_{\infty}+\delta\left(\left(T_{1}+\delta_{\infty}\right)-\delta_{\infty}\right) \leq 0
$$

and thus $T_{1}+\delta\left(T_{1}\right) \leq 0$. Thus, the event $\left(S+\delta_{\infty}, 1\right)$ gets removed from the output list and the output signal is the constant-zero signal.

Lemma 9. Let $c$ be a single-history channel with initial value 0. The following statements are equivalent:

1. c is not a constant-delay channel.

2. There exist a pulse s such that $c(s)$ is the zero signal.

3. $\gamma(c)>0$ 
Proof. Let $\delta$ be the delay function of $c$. If $s$ is a pulse of length $\Delta$, then $c(s)$ is zero if and only if

$$
\Delta-\delta_{\infty}+\delta\left(\Delta-\delta_{\infty}\right) \leq 0
$$

This implies $\gamma(c) \geq \Delta$ and hence establishes the equivalence of (2) and (3). If we can show that $c$ is not a constant-delay channel if and only if

$$
\exists \varepsilon>0: \delta\left(-\delta_{\infty}+\varepsilon\right) \leq \delta_{\infty}-\varepsilon,
$$

then we can choose $\Delta=\varepsilon$, concluding the proof.

The sufficiency of Eq. (6) for $c$ not being a constant-delay channel is immediate. To prove the necessity of Eq. (6), assume that $c$ is not a constant-delay channel. Then there exist $\beta, \beta^{\prime}>0$ such that $\delta\left(\beta-\delta_{\infty}\right)<$ $\delta\left(\beta^{\prime}-\delta_{\infty}\right)$ and since $\delta$ is nondecreasing, $\delta\left(\beta-\delta_{\infty}\right)<\delta_{\infty}$. Thus, there exists a $z>0$, such that,

$$
\delta\left(\beta-\delta_{\infty}\right) \leq \delta_{\infty}-z .
$$

There are two cases for $z$ : If $\beta \leq z$, we obtain from Eq. (77) that $\delta\left(\beta-\delta_{\infty}\right) \leq \delta_{\infty}-\beta$. Choosing $\varepsilon=\beta$ shows that Eq. (6) holds. Otherwise, i.e., if $\beta>z$, we obtain from Eq. (7) and the fact that $\delta$ is nondecreasing

$$
\delta\left(z-\delta_{\infty}\right) \leq \delta\left(\beta-\delta_{\infty}\right) \leq \delta_{\infty}-z .
$$

Choosing $\varepsilon=z$ shows that Eq. (6) holds.

Note that, while Lemmas 8 and 9 hold for both forgetful and non-forgetful single-history channels, the following lemma does fundamentally not hold for arbitrary non-forgetful channels.

Lemma 10. Let $c$ be a non-constant-delay forgetful single-history channel with initial value 0 . Let $s$ be a signal that does not contain pulses of length greater or equal to $\gamma(c)$ and that is not eventually equal to 1. Then $c(s)$ is the zero signal.

Proof. The lemma is proved by inductively repeating the proof of Lemma 8 for all pulses contained in $s$.

Lemma 11. Circuit $C_{\mathrm{ff}}$ solves bounded $S P F$.

Proof. We first note that, given an input signal, there is a unique execution for circuit $C_{\mathrm{ff}}$ according to Lemma 2, because the sole non-constant channel $c^{*}$ is not part of a feedback loop.

The well-formedness properties (F1) and (F2) of SPF are hence fulfilled. The non-generation property (F3) is also obvious.

If the input signal is a pulse of length at least $\varepsilon$, then $s_{\mathrm{OR}}(t)=1$ for all $t \geq S+1$, and hence $s_{o}(t)=1$ for all $t \geq S+1+\delta^{*}(\infty)$. In particular, this shows the nontriviality property (F4).

If the input signal is a pulse of length less than $\varepsilon$, then $s_{\mathrm{OR}}(t)$ only contains pulses of lengths less than $\varepsilon$, hence less than $\gamma\left(c^{*}\right)$ by the choice of $\varepsilon$. By Lemma 10, the output signal is zero in this case. This, together with the above, shows (F5) and (F6).

It remains to show that assuming $c^{*}$ to have initial value 0 is is not restricting: If its initial value is 1 we modify circuit $C_{\mathrm{ff}}$ by adding an inverter before and after channel $c^{*}$. A proof analogous to Lemma 11]s yields:

Theorem 3. Let $c^{*}$ be a non-constant-delay forgetful single-history channel. Then there exists a circuit solving bounded SPF whose channels are either constant-delay channels or $c^{*}$. 


\subsection{Non-Forgetful Channels}

Theorem 4 reveals that a single non-constant-delay non-forgetful single-history channel $c^{*}$ (with initial value 0) also allows to solve bounded SPF:

Theorem 4. Let $c^{*}$ be a non-constant-delay non-forgetful single history channel with initial value 0. Then there exists a circuit solving SPF whose channels are all either constant-delay channels or $c^{*}$.

Let $\delta$ be the delay function of $c^{*}$. Recall from Lemma 6 that $\delta_{\text {inf }}<\delta_{\infty}$, since $\delta$ is non-decreasing and not constant. We distinguish three cases for function $\delta$ with respect to its behavior at $-\delta_{\text {inf }}$.

1. There exists a $t>-\delta_{\text {inf }}$ such that $\delta(t)<\delta_{\infty}$.

2. $\delta(t)=\delta_{\infty}$ for all $t>-\delta_{\text {inf }}$, and

$2.1 \delta$ is continuous at $-\delta_{\text {inf }}$, i.e., at $-\delta_{\text {inf }}$ its left $\operatorname{limit}_{\lim _{t \rightarrow 0^{-}}} \delta\left(-\delta_{\text {inf }}+t\right)$ equals its right limit $\delta_{\infty}$.

$2.2 \delta$ is non-continuous at $-\delta_{\text {inf }}$, i.e., $\delta^{-}=\lim _{t \rightarrow 0^{-}} \delta\left(-\delta_{\text {inf }}+t\right)<\delta_{\infty}$.

For Cases 1 and 2.1, we show that circuit $C_{\mathrm{NF}}$ depicted in Fig. 11 solves bounded SPF. All its clocks $C L K_{A / C / F}$ produce a signal with period $A+B+C+D$, where parameters $A$ to $D$ are chosen later on in accordance with $\delta$. Let $\tau_{k}=k(A+B+C+D)$ denote the beginning of the $k$-th round, for $k \geq 0$. Clock $C L K_{C}$ is designed such that its output signal is 0 during $\left[\tau_{k}, \tau_{k}+A+B\right) \cup\left[\tau_{k}+A+B+C, \tau_{k+1}\right)$ and 1 during $\left[\tau_{k}+A+B, \tau_{k}+A+B+C\right)$. Such a clock can easily be built from constant-delay channels and inverters only. Clock $C L K_{A}$ 's output signal is 1 during $\left[\tau_{k}, \tau_{k}+A\right)$ and 0 during $\left[\tau_{k}+A, \tau_{k+1}\right)$. The output signal of $C L K_{F}$ is 0 during $\left[\tau_{k}, \tau_{k}+E\right) \cup\left[\tau_{k}+E+F, \tau_{k+1}\right)$ and 1 during $\left[\tau_{k}+E, \tau_{k}+E+F\right)$. Again, $E$ and $F$ are chosen later on in accordance with $\delta$.

Abbreviating $t_{k}=\tau_{k}+2$, we observe that circuit $C_{\mathrm{NF}}$ generates a signal $s_{O R}$ at the input of channel $c^{*}$, which is the OR of two subsignals that consist of four phases within time $\left[t_{k}, t_{k+1}\right), k \geq 0$ (i.e., per round): Phase $A$ (of round $k$ ) denotes the interval of times $\left[t_{k}, t_{k}+A\right.$ ), phase $B$ the interval $\left[t_{k}+A, t_{k}+A+B\right.$ ), phase $C$ the interval $\left[t_{k}+A+B, t_{k}+A+B+C\right)$ and phase $D$ the interval $\left[t_{k}+A+B+C, t_{k}+A+B+C+D\right)$. The value of $s_{O R}$ is 1 during phase $A$, and 0 during phases $B$ and $D$. During phase $C$ it is either 0 or contains a pulse, depending on signal $i$. Analogously, we define output phase $F$ (of round $k$ ) as the interval of times $\left[t_{k}+E, t_{k}+E+F\right)$. Note that phase $\mathrm{E}$ and $\mathrm{F}$ of round $k$ follow phase $\mathrm{D}$ of round $k$, and overlap with phase A of round $k+1$.

Informally, for Cases 1 and 2.1, circuit $C_{\mathrm{NF}}$ solves bounded SPF according to the following reasoning: Properties (F1) and (F2) trivially hold for circuit $C_{\mathrm{NF}}$. Clearly, if the circuit's input signal is 0 , then the channel's input signal $s_{O R}$ is 0 during phase $C$ of all rounds $k \geq 0$. Subsequently, we will prove that if this is the case, then the channel's output signal $c^{*}\left(s_{O R}\right)$ during phase $F$ is 0 for all rounds $k \geq 0$. Since phase $F$ is the only phase where $o$ could possibly produce a non-0 output due to the AND gate, both (F3) and (F5) follow. Property (F4) is implied by the fact that there exists an input signal $i$ such that $s_{O R}$ contains a pulse during phase $C$ of some round $k \geq 0$. We will prove below that if this is the case, then the channel's output signal is 1 during phase $F$ of round $k+1$. Essentially, this follows from a reduced delay of the rising transition at the end of phase D, caused by not forgetting the (cancelled) pulse in phase C. From this and the fact that all delays are bounded, (F6) follows.

Case 1. In this case, we choose

(i) $C>0, D>0$ and $0<\Delta<\delta_{\infty}$ such that $\delta\left(C+D-\delta_{\text {inf }}\right) \leq \delta_{\infty}-\Delta$. Such values for $C, D$ and $\Delta$ exist, because of the assumption of Case 1.

(ii) $\varepsilon>0, \varepsilon^{\prime}>0$ and $C>0$ small enough such that $\delta_{\infty}-\varepsilon^{\prime} \geq \delta_{\text {inf }}+\varepsilon+C$ and $\varepsilon^{\prime}<\Delta / 4$.

(iii) $C>0$ and $\varepsilon^{\prime}>0$ small enough such that $\delta\left(C+\varepsilon^{\prime}-\delta_{\infty}\right) \leq \delta_{\text {inf }}+\varepsilon$.

(iv) $A=B>\max \left(\varepsilon^{\prime}, \Delta, \delta_{\infty}-\delta_{\text {inf }}\right)$ and large enough such that $\delta\left(A-\delta_{\infty}\right) \geq \delta_{\infty}-\varepsilon^{\prime}$. 
(v) $E=\delta_{\infty}-\Delta$ and $F=\Delta / 2$.

It is easy to check that Assumptions (i)-(v) are compatible with each other.

Figures 9 and 10 depict signal $s_{O R}$ in absence and presence of a pulse. We will first show that the channel's output signal $c^{*}\left(s_{O R}\right)$ has value 0 during output phase $F$ of round 0 .

Proof. The signal is depicted in Fig. 9. Signal $s_{O R}$ 's transition to value 1 at time $t_{0}$ is delayed by $c^{*}$ by $\delta_{0}=\delta_{\infty}>0$. Its next transition back to value 0 at time $t_{0}+A$ is delayed by, say, $\delta_{1}$. Because of

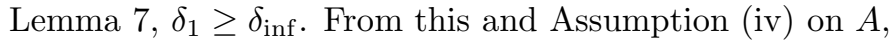

$$
A+\delta_{1}>\left(\delta_{\infty}-\delta_{\text {inf }}\right)+\delta_{\text {inf }}=\delta_{0} .
$$

It follows that output $c^{*}\left(s_{O R}\right)$ 's transition to 0 does not cancel $c^{*}\left(s_{O R}\right)$ 's transition to 1 from before. All of $s_{O R}$ 's following transitions occur at times at least $t_{0}+A+B$, and by (iv), at times greater than $t_{0}+\delta_{\infty}-\delta_{\text {inf }}$. Since all these transitions are delayed by at least $\delta_{\text {inf }}$ time, none of them can cancel $c^{*}\left(s_{O R}\right)$ 's transition to 1 at time $t_{0}+\delta_{\infty}$ either. Since channel $c^{*}$ has initial value 0 , it follows that its output has value 0 during $\left[0, t_{0}+\delta_{\infty}\right)$. Since

$$
t_{0}+\delta_{\infty}>t_{0}+\delta_{\infty}-\Delta / 2=t_{0}+E+F
$$

the channel's output indeed has value 0 during output phase $F$ of round 0 .

We next show, for $k \geq 0$, that if signal $s_{O R}$ does not contain a pulse within phase $C$ of round $k$, signal $c^{*}\left(s_{O R}\right)$ has value 0 during output phase $F$ of round $k+1$.

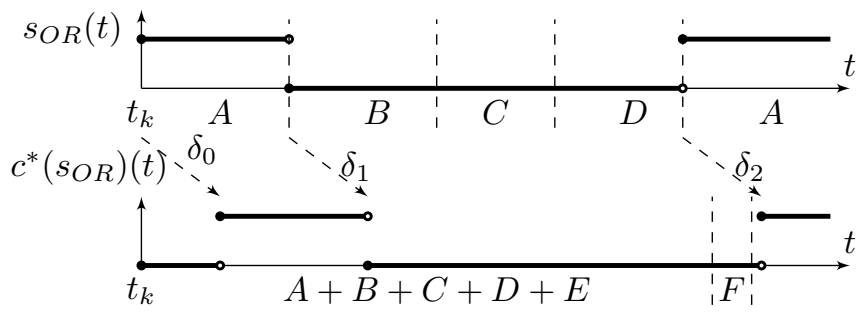

Figure 9: Case 1: Input and Output of channel $c^{*}$ in circuit $C_{\mathrm{NF}}$ if phase $C$ does not contain a pulse.

Proof. Assume the input signal $s_{O R}$ of channel $c^{*}$ does not contain a pulse within phase $C$ of round $k$. The signal is depicted in Fig. 9.

Signal $s_{O R}$ 's transition to value 1 at time $t_{k}$ is delayed by $c^{*}$ by $\delta_{0} \leq \delta_{\infty}$.

There is no transition of $s_{O R}$ before $s_{O R}$ 's transition back to value 0 at time $t_{k}+A$. Let $\delta_{1}$ be its delay. Because of (iv), and $\delta$ being non-decreasing, $A+\delta_{1}>\left(\delta_{\infty}-\delta_{\text {inf }}\right)+\delta_{\text {inf }}$. Thus, and because transitions are delayed by at least $\delta_{\text {inf }}$, none of the transitions from time $t_{k}+A$ on may cancel $c^{*}\left(s_{O R}\right)$ 's transition to 1 at time $t_{k}+\delta_{0}$.

The transition of $s_{O R}$ to value 1 at time $t_{k+1}=t_{k}+A+B+C+D$ is delayed by $\delta_{2}$, where

$$
\delta_{2}=\delta\left(B+C+D-\delta_{1}\right) \geq \delta\left(B-\delta_{\infty}\right) \geq \delta_{\infty}-\varepsilon^{\prime},
$$

because of Assumption (iv). Together with (ii) this yields

$$
\delta_{2}>\delta_{\infty}-\Delta / 4
$$

It will thus not occur at output $c^{*}\left(s_{O R}\right)$ before time $t_{k+1}+\delta_{\infty}-\Delta / 4$, and thus, by (v), not before the end of output phase $F$ of round $k+1$ at time $t_{k+1}+\delta_{\infty}-\Delta / 2$. 
Furthermore, from (8) and (iv),

$$
B+C+D+\delta_{2}>\delta_{\infty} \geq \delta_{1}
$$

because (iv) in particular implies $B>\varepsilon^{\prime}$. It follows that output $c^{*}\left(s_{O R}\right)$ 's transition to 1 does not cancel $c^{*}\left(s_{O R}\right)$ 's transition to 0 at time $t_{k}+A+\delta_{1}$. All $s_{O R}$ 's subsequent transitions occur at earliest at time $t_{k+1}+A>t_{k+1}+\delta_{\infty}-\delta_{\text {inf }}$, by (iv) and the fact that they are delayed by at least $\delta_{\text {inf }}$, hence cannot cancel $c^{*}\left(s_{O R}\right)$ 's transition to 1 at time $t_{k+1}+\delta_{2}$. Thus, $c^{*}\left(s_{O R}\right)$ has value 0 during $\left[t_{k}+A+\delta_{1}, t_{k+1}+\delta_{2}\right)$. Together with (9), this implies that $c^{*}\left(s_{O R}\right)$ 's value is 0 during phase $F$ of round $k+1$.

We now show, for $k \geq 0$, that if signal $s_{O R}$ contains a pulse within phase $C$ of round $k$, signal $c^{*}\left(s_{O R}\right)$ has value 1 during output phase $F$ of round $k+1$.

Proof. Assume the input signal $s_{O R}$ of channel $c^{*}$ contains a pulse within phase $C$ of round $k$. The signal is depicted in Fig. 10,

Signal $s_{O R}$ 's transition to value 1 at time $t_{k}$ is delayed by $\delta_{0} \leq \delta_{\infty}$. By the same arguments as in the proof before, it is not canceled by any following transition.

Signal $s_{O R}$ 's transition to 0 at time $t_{k}+A$ is delayed by $\delta_{1}$. Since no further transition of $s_{O R}$ occurs before time $t_{k}+A+B$, and since $B>\delta_{\infty}-\delta_{\text {inf }}$, it follows that $s_{O R}$ 's transition to 0 is not canceled by any following transition. The transition of $s_{O R}$ to 1 at time $t_{k}+A+u$ is delayed by $\delta_{2}$, where $\delta_{2}=\delta\left(u-\delta_{1}\right) \geq \delta\left(B-\delta_{\infty}\right)$, since $u \geq B, \delta_{1} \leq \delta_{\infty}$ and $\delta$ is non-decreasing. Thus, by (iv),

$$
\delta_{2} \geq \delta_{\infty}-\varepsilon^{\prime} .
$$

The transition of $s_{O R}$ back to value 0 at time $t_{k}+A+u+x$ is delayed by $\delta_{3}$, where

$$
\delta_{3}=\delta\left(x-\delta_{2}\right) \leq \delta\left(C+\varepsilon^{\prime}-\delta_{\infty}\right),
$$

since $x \leq C, \delta$ is non-decreasing, and by (10). By (iii),

$$
\delta_{3} \leq \delta_{\text {inf }}+\varepsilon .
$$

The pulse occurring during phase $C$ is filtered out at the output $c^{*}\left(s_{O R}\right)$ of channel $c^{*}$, since $\delta_{2} \geq x+\delta_{3}$ : The latter follows from (10), (ii) and (12), as $\delta_{2} \geq \delta_{\infty}-\varepsilon^{\prime} \geq \delta_{\text {inf }}+\varepsilon+C \geq \delta_{3}$.

The transition of $s_{O R}$ to value 1 at time $t_{k+1}=t_{k}+A+u+x+y$ is delayed by $\delta_{4}$, where $\delta_{4}=$ $\delta\left(y-\delta_{3}\right) \leq \delta\left(C+D-\delta_{\text {inf }}\right)$, since $\delta$ is non-decreasing and $y \leq C+D, \delta(t) \geq \delta_{\text {inf }}$ for all $t>-\delta_{\infty}$ such that $\delta_{3}=\delta\left(x-\delta_{2}\right) \geq \delta_{\text {inf }}$. By Assumption (i), we may thus deduce $\delta_{4} \leq \delta_{\infty}-\Delta$. Since no further transition of $s_{O R}$ occurs before time $t_{k+1}+A$, and $A>\delta_{\infty}-\delta_{\text {inf }}$ by Assumption (iv), $c^{*}\left(s_{O R}\right)$ 's transition at time $t_{k+1}+\delta_{4}$ is not canceled by any later transition. Since $A>\delta_{\infty}-\delta_{\mathrm{inf}}>E+F-\delta_{\mathrm{inf}}$, by Assumptions (iv) and (v), and the fact that a transition is delayed by at least time $\delta_{\text {inf }}$, no other transition of $c^{*}\left(s_{O R}\right)$ occurs during $\left(t_{k+1}+\delta_{4}, t_{k+1}+E+F\right]$. It follows that $c^{*}\left(s_{O R}\right)$ 's value is 1 during phase $F$ of round $k+1$.

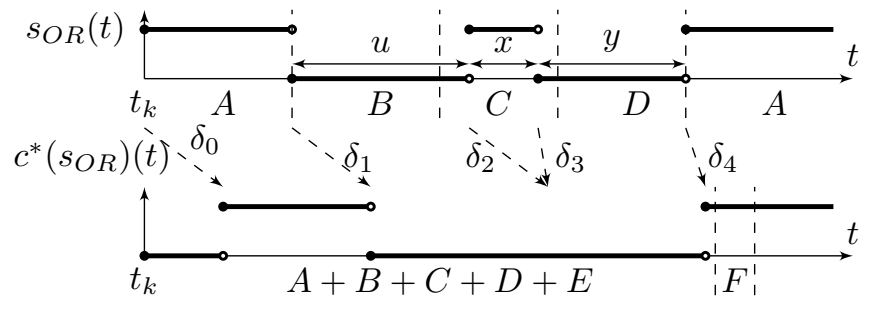

Figure 10: Case 1: Input and Output of channel $c^{*}$ in circuit $C_{\mathrm{NF}}$ if phase $C$ contains a pulse.

Case 2.1. In this case, we choose 


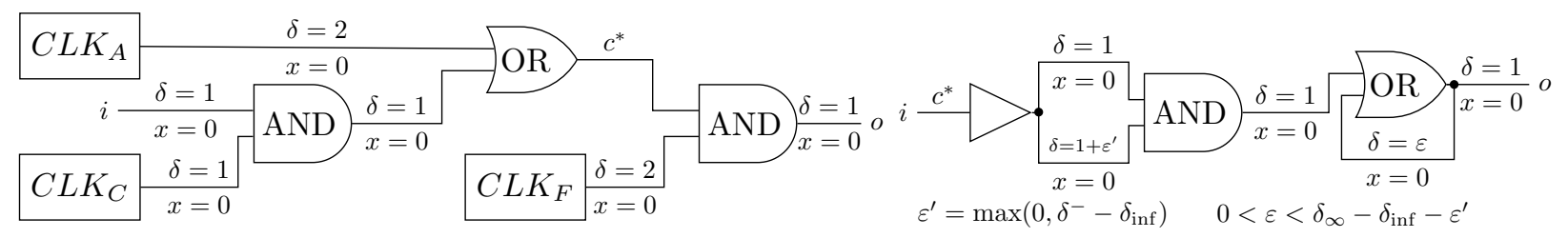

Figure 11: Circuit $C_{\mathrm{NF}}$ used in Cases 1 and 2.1.

Figure 12: Circuit $C_{\mathrm{NC}}$ used in Case 2.2.

(i) $A=D>\max \left(0, \delta_{\infty}-\delta_{\text {inf }}\right)$ and large enough such that $\delta\left(A-\delta_{\infty}\right)=\delta_{\infty}$. Such an $A$ must exist, because of the assumption of Case 2.1 .

(ii) $B, C, \varepsilon>0$ small enough such that $B+C+\varepsilon+\delta_{\text {inf }} \leq \delta_{\infty}$.

(iii) $0<\varepsilon^{\prime}<B+C$

(iv) $\varepsilon>0$ small enough such that $\delta\left(-\delta_{\text {inf }}-\varepsilon\right) \geq \delta_{\infty}-\varepsilon^{\prime}$. Such a value exists, since $\delta$ is continuous at $-\delta_{\text {inf }}$ by the assumption of Case 2.1 .

(v) $B+C>0$ small enough such that $\delta\left(B+C-\delta_{\infty}\right) \leq \delta_{\text {inf }}+\varepsilon$.

(vi) $E=A+\delta_{\infty}$ and $F=B+C-\varepsilon^{\prime}$.

Again, it is easy to verify that Assumptions (i)-(vi) are compatible with each other.

Figures 13 and 14 depict signal $s_{O R}$ in absence and presence of a pulse.

We next show by induction on $k \geq 0$ that signal $s_{O R}$ 's transition at time $t_{k}$ is delayed by $\delta_{\infty}$, and that the channel's output $c^{*}\left(s_{O R}\right)$ has value 0 during phase $F$ of round $k$ in the absence of a pulse within phase $C$ of round $k$, and value 1 in the presence of a pulse.

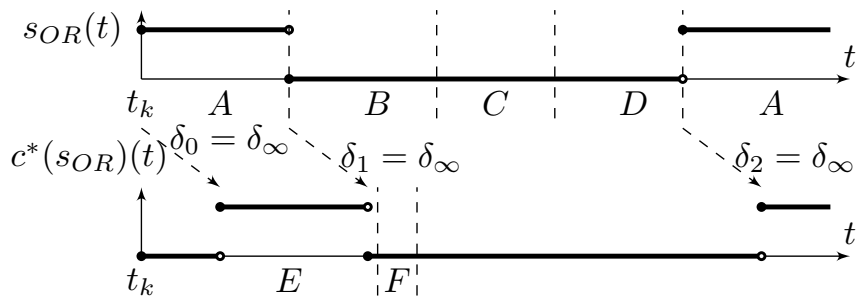

Figure 13: Case 2.1: Input and Output of channel $c^{*}$ in circuit $C_{\mathrm{NF}}$ if phase $C$ does not contain a pulse.

Proof. Assume the input signal $s_{O R}$ of channel $c^{*}$ contains no pulse within phase $C$ of round $k$. The signal is depicted in Fig. 13 .

Signal $s_{O R}$ 's transition to value 1 at time $t_{k}$ is delayed by some $\delta_{0}$. Clearly, if $k=0$ (i.e., in round 0 ), $\delta_{0}=\delta_{\infty}$. As induction hypothesis assume in the following that signal $s_{O R}$ 's transition at time $t_{k}$ is delayed by $\delta_{\infty}$. We will show that this implies that signal $s_{O R}$ 's transition at time $t_{k+1}$ is delayed by $\delta_{\infty}$.

Obviously, the next transition of $s_{O R}$ back to value 0 at time $t_{k}+A$ is delayed by $\delta_{1}$, where

$$
\delta_{1}=\delta\left(A-\delta_{0}\right)=\delta\left(A-\delta_{\infty}\right)=\delta_{\infty}
$$

by the choice of $A$ according to Assumption (i). Further, by Assumption (i), $A>\delta_{\infty}-\delta_{\text {inf }}$, implying that no transition of $s_{O R}$ after time $t_{k}$ can cancel the transition of $c^{*}\left(s_{O R}\right)$ to 1 at time $t_{k}+\delta_{0}$.

The transition of $s_{O R}$ to value 1 at time $t_{k+1}=t_{k}+A+B+C+D$ is delayed by $\delta_{2}$, where

$$
\delta_{2}=\delta\left(B+C+D-\delta_{1}\right)=\delta\left(B+C+D-\delta_{\infty}\right)=\delta_{\infty},
$$


because of Assumption (i). Thus, the initial transition of round $k+1$ at time $t_{k+1}$ will be delayed by $\delta_{\infty}$, which completes the inductive step. Since $D>\delta_{\infty}-\delta_{\text {inf }}>0$, by Assumption (i), it follows that $c^{*}\left(s_{O R}\right)$ 's transition to 0 at time $t_{k}+A+\delta_{1}$ is not canceled by any transition. By analogous arguments, the transition to 1 at time $t_{k+1}+\delta_{2}$ is not canceled by any transition. Our choice of $E$ and $F$ in (vi) thus implies that the channel output's value is 0 during phase $F$ of round $k$, see Fig. 13 .

Proof. Now assume that there is a pulse within phase $C$ of round $k$. The channel's input and output signals are depicted in Fig. 14.

Signal $s_{O R}$ 's initial transition to value 1 at time $t_{k}$ clearly is delayed by $\delta_{0}=\delta_{\infty}$ if $k=0$. As induction hypothesis assume in the following that $s_{O R}$ 's transition at time $t_{k}$ is delayed by $\delta_{\infty}$. We will show that this implies that $s_{O R}$ 's transition at time $t_{k+1}$ is delayed by $\delta_{\infty}$.

By the same reasoning as in the proof before, $c^{*}\left(s_{O R}\right)$ 's transition to 1 at time $t_{k}+\delta_{0}$ is not canceled by any following transition. Further, $s_{O R}$ 's transition back to value 0 at time $t_{k}+A$ is delayed by $\delta_{1}=\delta_{\infty}$.

The transition of $s_{O R}$ to value 1 at time $t_{k}+A+u$ is delayed by $\delta_{2}$, where $\delta_{2}=\delta\left(u-\delta_{1}\right) \leq \delta\left(B+C-\delta_{\infty}\right) \leq$ $\delta_{\text {inf }}+\varepsilon$, by Assumption (v). From (ii), we further obtain $u+\delta_{2} \leq B+C+\delta_{\text {inf }}+\varepsilon \leq \delta_{\infty}$. It follows that this output transition cancels the last output transition to 0 .

The transition of $s_{O R}$ back to value 0 at time $t_{k}+A+u+x$ is delayed by $\delta_{3}$, where $\delta_{3}=\delta\left(x-\delta_{2}\right) \geq$ $\delta\left(-\delta_{\text {inf }}-\varepsilon\right) \geq \delta_{\infty}-\varepsilon^{\prime}$, holds because of Assumption (iv).

The transition of $s_{O R}$ to value 1 at time $t_{k+1}$ is delayed by $\delta_{4}$, where $\delta_{4}=\delta\left(y-\delta_{3}\right) \geq \delta\left(D-\delta_{\infty}\right)=\delta_{\infty}$, by Assumption (i), which completes the inductive step.

Moreover, since $D>\delta_{\infty}-\delta_{\text {inf }}>0$, it follows that $c^{*}\left(s_{O R}\right)^{\prime}$ 's transition to 0 at time $t_{k}+A+u+x+\delta_{3}$ is not canceled by any transition. By similar arguments, $c^{*}\left(s_{O R}\right)$ 's transition to 1 at time $t_{k+1}+\delta_{4}$ is not canceled by any following transition.

Assumption (vi) hence implies that $c^{*}\left(s_{O R}\right)$ 's value is 1 during phase $F$ of round $k+1$, see Fig. 14.

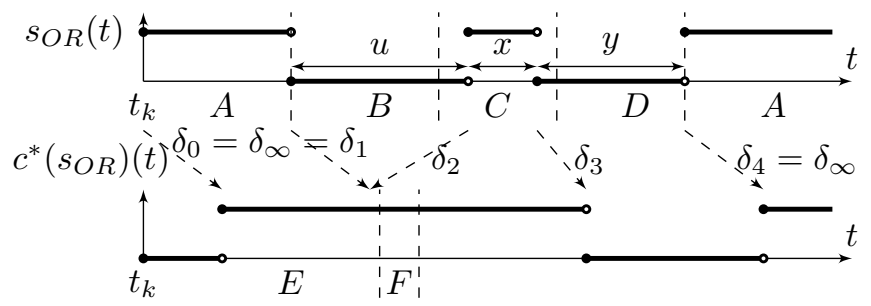

Figure 14: Case 2.1: Input and Output of channel $c^{*}$ in circuit $C_{\mathrm{NF}}$ if phase $C$ contains a pulse.

Case 2.2. For this case, circuit $C_{\mathrm{NC}}$ depicted in Fig. 12 solves bounded SPF. The algorithm and its proof rest on the following idea: We first show in Lemma 12 that every channel $c^{*}$ whose $\delta$ is in accordance with Case 2.2 does not produce pulses of length within the non-zero interval $\left[\max \left(0, \delta^{-}-\delta_{\text {inf }}\right), \delta_{\infty}-\delta_{\text {inf }}\right)$. The remaining part of circuit $C_{\mathrm{NC}}$ thus just has to filter out all pulses with duration less than $\max \left(0, \delta^{-}-\delta_{\text {inf }}\right)$ (ensured by the AND gate) and continuously hold all pulses of length $\delta_{\infty}-\delta_{\text {inf }}$ (done by the OR gate).

Lemma 12. Let $c^{*}$ be a non-constant-delay non-forgetful channel chosen in accordance to Case 2.2. If the channel's input signal is a pulse, then its output signal is either 0 or a pulse whose length is not within the non-zero interval $\left[\max \left(0, \delta^{-}-\delta_{\mathrm{inf}}\right), \delta_{\infty}-\delta_{\mathrm{inf}}\right]$.

Proof. Assume that $\delta\left(-\delta_{\text {inf }}\right)=\delta_{\infty}$; the proof for the case $\delta\left(-\delta_{\text {inf }}\right)=\delta^{-}<\delta_{\infty}$ is almost the same. Without loss of generality, assume that the input pulse starts at time 0 and let $x>0$ be its length. Clearly, the transition of the output signal to 1 is scheduled at time $\delta_{\infty}$, the transition back to 0 is scheduled at time $x+\delta\left(x-\delta_{\infty}\right)$. We distinguish two cases for the input pulse length $x$ :

In case $x<\delta_{\infty}-\delta_{\text {inf }}$, we have $\delta\left(x-\delta_{\infty}\right) \leq \delta^{-}$and the following two sub-cases: If additionally $x \leq \delta_{\infty}-\delta^{-}$, then $x+\delta\left(x-\delta_{\infty}\right) \leq x+\delta^{-} \leq \delta_{\infty}$, so the output events cancel. If $\delta_{\infty}-\delta_{\text {inf }}>x>\delta_{\infty}-\delta^{-}$, the length 


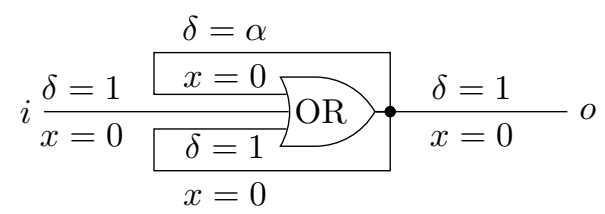

Figure 15: Circuit $C_{\mathrm{ev}}$ solving eventual SPF.

of the output pulse is $x+\delta\left(x-\delta_{\infty}\right)-\delta_{\infty}<\delta^{-}-\delta_{\text {inf }}$. This confirms the lower boundary of the "forbidden pulse length interval" given in our lemma. In case of $x \geq \delta_{\infty}-\delta_{\text {inf }}$, on the other hand, $\delta\left(x-\delta_{\infty}\right)=\delta_{\infty}$ a pulse with length $x+\delta\left(x-\delta_{\infty}\right)-\delta_{\infty} \geq \delta_{\infty}-\delta_{\text {inf }}$ is generated at the output of $c^{*}$, which also confirms the upper boundary of the interval.

If we choose the circuit parameters in Fig. 12 according to $\varepsilon^{\prime}=\max \left(0, \delta^{-}-\delta_{\text {inf }}\right)$ and $0<\varepsilon<\delta_{\infty}-\delta_{\text {inf }}-\varepsilon^{\prime}$, it is not difficult to show that the resulting circuit $C_{\mathrm{NC}}$ solves bounded SPF in Case 2.2: Properties (F1) to (F3) trivially hold for circuit $C_{\mathrm{NC}}$. To prove (F4), consider that if the input signal $i$ is a pulse of length $2 \delta_{\infty}$, the output signal $s_{c^{*}(i)}$ of $c^{*}$ is a pulse of length at least $\delta_{\infty}$. Thus, the output of the AND gate $s_{A N D}$ is a pulse of length at least $\delta_{\infty}-\varepsilon^{\prime}>\varepsilon$, resulting in the circuit's output $o$ making a transition to 1 and remaining 1 from there on.

Property (F5) directly follows from Lemma 12, If $s_{c^{*}(i)}$ is a pulse of length smaller than $\max \left(0, \delta^{-}-\delta_{\text {inf }}\right)=$ $\varepsilon^{\prime}$, then it is completely filtered out; $s_{A N D}$ and hence $o$ are hence permanently 0. Otherwise, by Lemma 12. $s_{c^{*}(i)}$ must be a pulse of length at least $\delta_{\infty}-\delta_{\text {inf }}$. Thus, $s_{A N D}$ is a pulse of length at least $\delta_{\infty}-\delta_{\text {inf }}-\varepsilon^{\prime}>\varepsilon$, which is sufficiently long to be permanently captured in the storage looped formed by the OR gate. The circuit's output $o$ hence makes a transition to 1 and remains 1 from there on.

Finally, (F6) is due to bounded channel delays.

\section{Eventual Short-Pulse Filtration with Constant Delays}

We proved that SPF is not solvable with constant-delay channels. In this section, we consider the weaker eventual SPF problem, which drops the "no short pulses" requirement (F5) and replaces it with its eventual analogon (F5e). We show that eventual SPF is solvable using only constant-delay channels. More specifically, we prove that circuit $C_{\text {ev }}$ in Fig. 15] solves eventual SPF. The circuit contains a delay parameter $\alpha$, which we will choose to be a positive irrational like $\alpha=\sqrt{2}$.

We will show that the circuit's output is eventually stable at 1 whenever the input is a pulse of positive length. We derive a bound on this stabilization time in terms of the input pulse length $\Delta$. The bound is almost linear in $1 / \Delta$ : It is in the order of $O\left(\Delta^{-1-\varepsilon}\right)$ for all $\varepsilon>0$.

The measure points of circuit $C_{\mathrm{ev}}$ for time $t$ are of the form $t-(\alpha k+\ell)-2$, where $k$ and $\ell$ are nonnegative integers. We can hence characterize the circuit's behavior with the following obvious lemma.

Lemma 13. In every execution $\left(s_{v}\right)$ of circuit $C_{\mathrm{ev}}$, the following are equivalent: (i) $s_{o}(t)=1$, and (ii) there exist nonnegative integers $k$ and $\ell$ such that $s_{i}(t-(\alpha k+\ell)-2)=1$.

We may restrict our considerations to input pulses starting at time 0 . In the following, let the input signal $s_{i}$ be a pulse of length $\Delta>0$. We are looking for the stabilization time, which is the minimal time $T=T(\Delta)$ such that, for all $t \geq T$, we have $s_{o}(t)=1$.

To prove finiteness and effective bounds on the stabilization time, we relate it to the number-theoretic concept of discrepancy of the sequence $(\alpha n)$ modulo 1 (see, e.g., 10]). The discrepancy compares the number of sequence elements in a given interval with their expected number if the elements were uniformly distributed.

For a given nonempty subinterval $(x, y]$ of $(0,1]$ and a given positive integer $N$, let $A(x, y ; N)$ denote the number of $\alpha n$ 's with $n \leq N$ that lie in the interval modulo $1: \alpha n \in(x, y]+\mathbb{Z}$. The expected number of such 
$\alpha n$ 's is $(y-x) N$. The discrepancy $D_{N}(\alpha)$ is then defined as the maximum difference between $A(x, y ; N)$ and $(y-x) N$, formed over all nonempty subintervals $(x, y]$ of $(0,1]$.

It is well-known that $D_{N}(\alpha) / N \rightarrow 0$ if and only if $\alpha$ is irrational. Also, if $\alpha$ has a bounded continued fraction expansion, then $D_{N}(\alpha)=O(\log N)$ and the constant can be computed [20. This is, in particular, true for $\alpha=\sqrt{2}$.

Lemma 14. Let $K=K(\Delta)$ be the least integer $K$ such that for all real there exists an integer $k, 0 \leq k \leq K$, with $\alpha k \in(t-\Delta, t]+\mathbb{Z}$. Then, $T(\Delta) \leq \alpha \cdot K(\Delta)+\Delta+2$.

Proof. The lemma is trivial if $K=\infty$, so assume the contrary.

Let $t \geq \alpha K+\Delta+2$. By the definition of $K$, there exists a $k$ with $0 \leq k \leq K$ and an $\ell$ such that $t-\Delta-\ell-2<\alpha k \leq t-\ell-2$, which is equivalent to $0 \leq t-(\alpha k+\ell)-2<\Delta$.

By Lemma 13, it remains to prove that $\ell$ is nonnegative. The inequality $t-(\alpha k+\ell)-2<\Delta$ is equivalent to $\ell>t-\Delta-\alpha k-2$. Noting $-\alpha k \geq-\alpha K$ and $t \geq \alpha K+\Delta+2$ shows $\ell>0$ and concludes the proof.

Lemma 15. Let $0<\Delta \leq 1$. If $D_{N}(\alpha) / N<\Delta / 2$, then $K(\Delta) \leq N$.

Proof. Suppose the contrary, i.e., that there exists a real $t$ such that, for all $n \leq N$, we have $\alpha n \notin(t-\Delta, t]+\mathbb{Z}$. Let $0<x<y \leq z<u \leq 1$ such that we can decompose the interval $(t-\Delta, t]+\mathbb{Z}=((x, y]+\mathbb{Z}) \cup((z, u]+\mathbb{Z})$ modulo 1. None of the two intervals $(x, y]$ and $(z, u]$ contains an $\alpha n$ modulo 1 with $n \leq N$. Hence $A(x, y ; N)=A(u, z ; N)=0$, which implies $2 D_{N}(\alpha) \geq(y-x) N+(u-z) N=\Delta N$, a contradiction.

Theorem 5. Circuit $C_{\mathrm{ev}}$ solves eventual SPF if $\alpha$ is irrational. If $\alpha=\sqrt{2}$, the stabilization time satisfies $T(\Delta)=O\left(\Delta^{-1-\varepsilon}\right)$ as $\Delta \rightarrow 0$ for all $\varepsilon>0$.

Proof. (F1) and (F2) are obviously fulfilled. Because all initial values of channels are 0, also (F3) holds. Because $D_{N}(\alpha) / N \rightarrow 0$ whenever $\alpha$ is irrational, for all $\Delta>0$, there exists some $N$ such that $D_{N}(\alpha) / N<$ $\Delta / 2$. Hence Lemma 15 and Lemma 14 show that $T(\Delta)$ is finite, which shows (F4) and (F5e).

We now prove the bound on the stabilization time. Let $\gamma=-1-\varepsilon<-1$. There exists a $C_{1}>0$ such that $D_{N}(\alpha) \leq C_{1} \log N$. Because $1+1 / \gamma>0$, there exists a $C_{2}>0$ such that $\log N<C_{2} N^{1+1 / \gamma}$. Thus if

$$
N \geq\left(\frac{\Delta}{2 C_{1} C_{2}}\right)^{\gamma}
$$

then

$$
\frac{D_{N}(\alpha)}{N} \leq \frac{C_{1} \log N}{N}<C_{1} C_{2} N^{1 / \gamma} \leq \frac{\Delta}{2}
$$

which, by Lemma 15, implies

$$
K(\Delta) \leq\left(\frac{\Delta}{2 C_{1} C_{2}}\right)^{\gamma}+1
$$

for all $0<\Delta \leq 1$. That is, $K(\Delta)=O\left(\Delta^{\gamma}\right)$ as $\Delta \rightarrow 0$.

It is easy to see that $K(\Delta) \rightarrow \infty$ as $\Delta \rightarrow 0$. Hence Lemma 14 implies $T(\Delta)=O(K(\Delta))$ as $\Delta \rightarrow 0$ as asserted.

\section{Conclusion}

We showed that binary circuit models using bounded single-history channels, hence all binary models known to date, fail to faithfully model glitch propagation: In case of constant-delay channels, SPF turned out to be unsolvable, which is in contradiction to physical reality. In case of non-constant-delay channels, even bounded SPF is solvable, again in contradiction to physical reality. Future binary models aiming at faithful glitch propagation modeling hence cannot have the bounded single-history property.

We hope that our results provide a signpost for future research on adequate binary circuit models: As confirmed by the fact that the weaker eventual SPF problem is already solvable with constant-delay 
channels, SPF is well suited for capturing the peculiarities of glitch propagation while not being overly restrictive. Moreover, in the proofs of our core results, we actually used weaker properties than actually guaranteed by single-history channels. It may hence be possible to re-use part of those for alternative weaker channel models.

\section{References}

[1] P.J. Ashenden, The Designer's Guide to VHDL, 3rd ed., Morgan Kaufmann, 2008.

[2] J.C. Barros and B.W. Johnson, "Equivalence of the Arbiter, the Synchronizer, the Latch, and the Inertial Delay," IEEE Trans. Computers, vol. 32, no. 7, pp. 603-614, July 1983.

[3] M.J. Bellido-Díaz et al., "Logical Modelling of Delay Degradation Effect in Static CMOS Gates," IEE Proc. Circuits, Devices, and Systems, vol. 147, no. 2, pp. 107-117, Apr. 2000.

[4] M.J. Bellido-Díaz, J. Juan-Chico, and M. Valencia, Logic-Timing Simulation and the Degradation Delay Model. London: Imperial College Press, 2006.

[5] M.S. Branicky, "Universal Computation and Other Capabilities of Hybrid and Continuous Dynamical Systems," Theoretical Computer Science, vol. 138, no. 1, pp. 67-100, Feb. 1995.

[6] J.A. Brzozowski and J.C. Ebergen, "On the Delay-Sensitivity of Gate Networks," IEEE Trans. Computers, vol. 41, no. 11, pp. 1349-1360, Nov. 1992.

[7] C. Constantinescu, "Trends and Challenges in VLSI Circuit Reliability," IEEE Micro, vol. 23, no. 4, pp. 14-19, July 2003.

[8] D. Dolev et al., "Fault-Tolerant Algorithms for Tick-Generation in Asynchronous Logic: Robust Pulse Generation (Extended Abstract)," Proc. 13th Int'l Symp. Stabilization, Safety, and Security of Distributed Systems (SSS'11), LNCS 6976, Springer, pp. 163-177, Oct. 2011.

[9] S. Dolev, Self-Stabilization, MIT Press, 2000.

[10] M. Drmota and R.F. Tichy, Sequences, Discrepancies, and Applications, Springer, 1997.

[11] G. Fuchs, M. Függer, and A. Steininger, "On the Threat of Metastability in an Asynchronous FaultTolerant Clock Generation Scheme," Proc. 15th IEEE Int'l Symp. Asynchronous Circuits and Systems (ASYNC'09), pp. 127-136, May 2009.

[12] M. Függer and U. Schmid, "Reconciling Fault-Tolerant Distributed Computing and Systems-on-Chip", Distributed Computing 24(6), 2012, pp. 323-355.

[13] M.J. Gadlage et al., "Digital Device Error Rate Trends in Advanced CMOS Technologies," IEEE Trans. Nuclear Science, vol. 53, no. 6, pp. 3466-3471, Dec. 2006.

[14] D.J. Kinniment, Synchronization and Arbitration in Digital Systems, Wiley, 2007.

[15] L.R. Marino, "The Effect of Asynchronous Inputs on Sequential Network Reliability," IEEE Trans. Computers, vol. 26, no. 11, pp. 1082-1090, Nov. 1977.

[16] L.R. Marino, "General Theory of Metastable Operation," IEEE Trans. Computers, vol. 30, no. 2, pp. 107-115, Feb. 1981.

[17] M.S. Maza and M.L. Aranda, "Analysis of Clock Distribution Networks in the Presence of Crosstalk and Groundbounce," Proc. 8th Int'l IEEE Conf. Electronics, Circuits, and Systems (ICECS'01), pp. 773776, Sep. 2001. 
[18] M. Mendler and T. Stroup, "Newtonian Arbiters Cannot Be Proven Correct," Formal Methods in System Design, vol. 3, no. 3, pp. 233-257, Dec. 1993.

[19] F.U. Rosenberger et al., "Q-Modules: Internally Clocked Delay-Insensitive Modules," IEEE Trans. Computers, vol. 37, no. 9, pp. 1005-1018, Sep. 1988.

[20] J. Schoissengeier, "The Discrepancy of $(n \alpha)_{n \geq 1}$," Mathematische Annalen, vol. 296, no. 1, pp. 529-545, Dec. 1993.

[21] I.E. Sutherland, "Micropipelines," Comm. ACM, vol. 32, no. 6, pp. 720-738, June 1989.

[22] S.H. Unger, "Asynchronous Sequential Switching Circuits with Unrestricted Input Changes," IEEE Trans. Computers, vol. 20, no. 12, pp. 1437-1444, Dec. 1971. 